\title{
ON THE MINIMIZATION OF SOME NONCONVEX DOUBLE OBSTACLE PROBLEMS
}

\section{A. ELFANNI}

Received 11 October 2002

We consider a nonconvex variational problem for which the set of admissible functions consists of all Lipschitz functions located between two fixed obstacles. It turns out that the value of the minimization problem at hand is equal to zero when the obstacles do not touch each other; otherwise, it might be positive. The results are obtained through the construction of suitable minimizing sequences. Interpolating these minimizing sequences in some discrete space, a numerical analysis is then carried out.

\section{Introduction}

Let $\Omega$ denote a bounded and convex domain in $\mathbb{R}^{n}(n \geq 2)$ of boundary $\partial \Omega$ and of closure $\bar{\Omega}$. Let $\varphi: \mathbb{R}^{n} \rightarrow \mathbb{R}$ be a continuous function such that

$$
\begin{gathered}
\varphi\left(w_{i}\right)=0 \quad \forall i=1, \ldots, p, \\
\varphi(w)>0 \quad \forall w \neq w_{i}, i=1, \ldots, p,
\end{gathered}
$$

where $w_{i}$ 's are $p$ elements of $\mathbb{R}^{n}(p \geq 2)$. For instance, in solid-solid phase transformations, the function $\varphi$ could be some elastic energy that vanishes at wells $w_{i}^{\prime}$ 's. These wells stand for the stress-free states of a body represented by $\Omega$. For further details about the physical background and also the mathematical modelling, we refer the reader to $[2,3]$ and the references quoted therein. We assume that, for some physical reasons (e.g., some loads applied to the body $\Omega$ ), the deformations are constrained not to exceed some fixed obstacles. To make precise the formulation of our 
problem, let $\alpha, \beta$, and $G$ be three Lipschitz continuous functions, that is,

$$
\alpha, \beta, G \in W^{1, \infty}(\Omega)
$$

such that

$$
\alpha(x) \leq G(x) \leq \beta(x) \text { in } \Omega .
$$

Here $W^{1, \infty}(\Omega)$ is the usual Sobolev space of all weakly differentiable functions $u: \Omega \rightarrow \mathbb{R}$ such that $u$ and $|\nabla u|$ are essentially bounded. For a further discussion of Sobolev spaces, we refer the reader to [1]. We denote by $\mathcal{K}$ the following set:

$\mathcal{K}(\Omega):=\mathcal{K}=\left\{v \in W^{1, \infty}(\Omega): \alpha(x) \leq v(x) \leq \beta(x)\right.$ in $\Omega, v(x)=G(x)$ on $\left.\partial \Omega\right\}$.

Then we consider the following problem:

$$
I:=\inf _{v \in \mathcal{K}} \int_{\Omega} \varphi(\nabla v(x)) d x .
$$

More precisely, we consider the case when

$$
\nabla G(x) \in \operatorname{Co}\left(w_{i}\right) \text { a.e. in } \Omega,
$$

where $\operatorname{Co}\left(w_{i}\right)$ denotes the convex hull of the wells $w_{i}$ 's.

For the numerical purpose and in order to simplify, we will assume that $\Omega$ is polyhedral. Let $\left(\tau_{h}\right)$ be a regular family of triangulations of $\Omega$ (see [11]). This means that

$$
\forall h>0 \quad\left\{\begin{array}{l}
\forall K \in \tau_{h}, K \text { is an } N \text {-simplex, } \\
\max _{K \in \mathcal{\tau}_{h}}\left(h_{K}\right)=h, \\
\exists v>0 \text { such that } \forall K \in \mathcal{\tau}_{h}, \frac{h_{K}}{\rho_{K}} \leq v,
\end{array}\right.
$$

where $h_{K}$ is the diameter of the $N$-simplex $K$ and $\rho_{K}$ its roundness (i.e., the largest diameter of the balls that could fit in $K)$. If $P_{1}(K)$ is the space of polynomials of degree 1 on $K$, we set

$$
\begin{aligned}
V_{h} & =\left\{v: \Omega \longrightarrow \mathbb{R} \text { continuous, } v / K \in P_{1}(K), \forall K \in \tau_{h}\right\}, \\
\mathcal{K}_{h} & :=\left\{v \in V_{h}: \alpha(p) \leq v(p) \leq \beta(p) \forall p \in \Sigma_{h}^{0}, v(p)=G(p) \forall p \in \Sigma_{h} \cap \partial \Omega\right\},
\end{aligned}
$$


where

$$
\begin{aligned}
& \Sigma_{h}=\left\{p \in \bar{\Omega} \mid p \text { is a vertex of } K \in \tau_{h}\right\}, \\
& \Sigma_{h}^{0}=\left\{p \in \Sigma_{h} \mid p \notin \partial \Omega\right\} .
\end{aligned}
$$

Then we denote by $I_{h}$ the following approximate version of (1.6):

$$
I_{h}:=\inf _{\mathcal{K}_{h}} \int_{\Omega} \varphi(\nabla v(x)) d x
$$

The (double) obstacle problems were intensively studied by many authors in the case of convex variational problems, that is, the function $\varphi$ in problem (1.6) is assumed to be convex. In this paper, we would like to investigate such problems in the presence of nonconvexity. Recall that the question of existence or nonexistence of minimizers for scalar noncovex variational problems with homogeneous boundary conditions is closed (see [10]). One can also see [9] for a contribution in this direction. The case of nonhomogeneous boundary conditions was first studied by Chipot (see [5]) and then other results were obtained in [6, 7]. The main concern of our paper is to compute the value of $I$ and obtain estimates for $\left|I_{h}-I\right|$ in terms of $h$. We denote by $\Omega^{\prime}$ the subset of $\Omega$ where the obstacles do not touch each other, that is,

$$
\Omega^{\prime}:=\{x \in \Omega: \alpha(x)<\beta(x)\} .
$$

Roughly speaking, our main results are summarized in what follows. Under some assumptions, one has

$$
I:=\inf _{v \in \mathcal{K}} \int_{\Omega} \varphi(\nabla v(x)) d x=\int_{\Omega \backslash \Omega^{\prime}} \varphi(\nabla G(x)) d x .
$$

Moreover, if $\left|\Omega \backslash \Omega^{\prime}\right|=0\left(\left|\Omega \backslash \Omega^{\prime}\right|\right.$ is the Lebesgue measure of $\left.\Omega \backslash \Omega^{\prime}\right)$, one has, for $h$ small enough,

$$
0 \leq I_{h} \leq C h^{1 / 2}
$$

where $C$ is a constant independent of $h$.

The plan of our paper will be as follows. In Section 2, we present all the intermediate results that we will need to prove our main results which are exposed in Section 3. We will try as far as possible to have our paper self-contained. 


\section{Preliminary results}

In this section, we collect all the ingredients which will be useful to prove our results. First we have the following lemma.

LEMMA 2.1. If $G$ is a Lipschitz continuous function satisfying (1.7), then there exists a Lipschitz function $\tilde{G}$ defined in $\mathbb{R}^{n}$ such that

$$
\tilde{G}=G \quad \text { in } \Omega \text {. }
$$

Moreover,

$$
\bigwedge_{i=1}^{p} w_{i} \cdot(x-y) \leq \tilde{G}(x)-\tilde{G}(y) \leq \bigvee_{i=1}^{p} w_{i} \cdot(x-y) \quad \forall x, y \in \mathbb{R}^{n}
$$

where $\bigwedge$ and $\bigvee$ denote, respectively, the infimum and supremum of functions and $a \cdot b$ is the scalar product of $a, b \in \mathbb{R}^{n}$.

Proof. Applying the mean value theorem after regularization (see [8] for details), one has

$$
G(x)-G(y) \geq \bigwedge_{i=1}^{p} w_{i} \cdot(x-y) \quad \text { for } x, y \in \Omega
$$

Then let

$$
\tilde{G}(x)=\inf _{y \in \Omega}\left\{G(y)-\bigwedge_{i=1}^{p} w_{i} \cdot(y-x)\right\}, \quad x \in \mathbb{R}^{n} .
$$

It is clear that $\tilde{G}(x) \leq G(x)$ in $\Omega$. Moreover, using (2.3), one has

$$
\tilde{G}(x) \geq G(x) \text { in } \Omega,
$$

so that

$$
\tilde{G}(x)=G(x) \text { in } \Omega .
$$

We now prove that

$$
\bigwedge_{i=1}^{p} w_{i} \cdot(x-y) \leq \tilde{G}(x)-\tilde{G}(y) \leq \bigvee_{i=1}^{p} w_{i} \cdot(x-y) \quad \text { for } x, y \in \mathbb{R}^{n} .
$$


For every $x, y \in \mathbb{R}^{n}$, one has

$$
\tilde{G}(x)=\inf _{z \in \Omega}\left\{G(z)-\bigwedge_{i=1}^{p} w_{i} \cdot(z-y)+\bigwedge_{i=1}^{p} w_{i} \cdot(z-y)-\bigwedge_{i=1}^{p} w_{i} \cdot(z-x)\right\}
$$

Since

$$
\bigwedge_{i=1}^{p} w_{i} \cdot(z-y)-\bigwedge_{i=1}^{p} w_{i} \cdot(z-x) \geq \bigwedge_{i=1}^{p} w_{i} \cdot(x-y)
$$

one gets

$$
\tilde{G}(x) \geq \inf _{z \in \Omega}\left\{G(z)-\bigwedge_{i=1}^{p} w_{i} \cdot(z-y)\right\}+\bigwedge_{i=1}^{p} w_{i} \cdot(x-y)
$$

that is,

$$
\tilde{G}(x)-\tilde{G}(y) \geq \bigwedge_{i=1}^{p} w_{i} \cdot(x-y) .
$$

Moreover, for every $x, y \in \mathbb{R}^{n}$, one has

$$
\bigvee_{i=1}^{p} w_{i} \cdot(x-y)=-\bigwedge_{i=1}^{p} w_{i} \cdot(y-x)
$$

Then the second inequality in (2.7) follows easily using the first inequality proved above. This completes the proof of the lemma.

Now let $\left(v_{1}, \ldots, v_{q}\right)$ be a basis of the space $W$ spanned by the wells $w_{i}$ 's and denote by $x_{z}$ the points of the lattice of size $h^{1 / 2}$ spanned by the $v_{i}^{\prime}$, that is, for any $z=\left(z_{1}, \ldots, z_{q}\right) \in \mathbb{Z}^{q}$, set

$$
x_{z}=\sum_{i=1}^{q} z_{i} h^{1 / 2} v_{i}
$$

Then we define the functions $\Lambda_{h}$ and $V_{h}$ by

$$
\begin{aligned}
& \Lambda_{h}(x)=\bigvee_{z \in \mathbb{Z}^{q}}\left(\bigwedge_{i=1}^{p} w_{i} \cdot\left(x-x_{z}\right)+\tilde{G}\left(x_{z}\right)\right), \\
& V_{h}(x)=\bigwedge_{z \in \mathbb{Z}^{q}}\left(\bigvee_{i=1}^{p} w_{i} \cdot\left(x-x_{z}\right)+\tilde{G}\left(x_{z}\right)\right) .
\end{aligned}
$$


By a unit cell of the lattice spanned by the $h^{1 / 2} v_{i}$ 's we mean a set of the type

$$
C_{z}=x_{z}+\left\{\sum_{i=1}^{q} \beta_{i} h^{1 / 2} v_{i} \mid \beta_{i} \in[0,1]\right\} .
$$

Then one has the following lemma.

LeMma 2.2. Denote by $C_{z_{0}}$ a unit cell spanned by $h^{1 / 2} v_{i}$ 's and by $E$ the set

$$
E=\left\{z \in \mathbb{Z}^{q} \mid z_{i}=0 \text { or } 1 \forall i=1, \ldots, q\right\}
$$

Assume that $G$ verifies (1.7); if $\operatorname{dim}(W)>1$, assume in addition that the family $\left(w_{p}-w_{1}, \ldots, w_{p}-w_{p-1}\right)$ is a basis of $W$ and $\left(v_{1}, \ldots, v_{p-1}\right)$ is its dual, where $\operatorname{dim}(W)$ denotes the dimension of $W$. Then,

$$
\begin{aligned}
& \Lambda_{h}(x)=\bigvee_{z^{\prime} \in z_{0}+E}\left(\bigwedge_{i=1}^{p} w_{i} \cdot\left(x-x_{z^{\prime}}\right)+\tilde{G}\left(x_{z^{\prime}}\right)\right), \\
& V_{h}(x)=\bigwedge_{z^{\prime} \in z_{0}+E}\left(\bigvee_{i=1}^{p} w_{i} \cdot\left(x-x_{z^{\prime}}\right)+\tilde{G}\left(x_{z^{\prime}}\right)\right) .
\end{aligned}
$$

Proof. We give here an astute proof for the case when $W$ is a one-dimensional space. This case was not treated in [6] where a technical proof for higher dimensions is given. Let $z_{0}, z \in \mathbb{Z}$ and $x \in C_{z_{0}}:=\left[x_{z_{0}}, x_{z_{0}+1}\right]$. One has either $x_{z_{0}} \in\left[x_{z}, x\right]$ or $x_{z_{0}+1} \in\left[x_{z}, x\right]$. We assume that $x_{z_{0}} \in\left[x_{z}, x\right]$; the other case can be handled similarly. There exists $\lambda \in[0,1]$ such that

$$
x_{z_{0}}=\lambda x_{z}+(1-\lambda) x
$$

Therefore

$$
x-x_{z_{0}}=\lambda\left(x-x_{z}\right), \quad x_{z_{0}}-x_{z}=(1-\lambda)\left(x-x_{z}\right) .
$$

One has

$$
\begin{aligned}
\bigwedge_{i=1}^{p} w_{i} \cdot( & \left.x-x_{z_{0}}\right)+\tilde{G}\left(x_{z_{0}}\right) \\
& =\bigwedge_{i=1}^{p} w_{i} \cdot\left(x-x_{z_{0}}\right)+\tilde{G}\left(x_{z_{0}}\right)-\tilde{G}\left(x_{z}\right)+\tilde{G}\left(x_{z}\right)
\end{aligned}
$$


so that

$$
\bigwedge_{i=1}^{p} w_{i} \cdot\left(x-x_{z_{0}}\right)+\tilde{G}\left(x_{z_{0}}\right) \geq \bigwedge_{i=1}^{p} w_{i} \cdot\left(x-x_{z_{0}}\right)+\bigwedge_{i=1}^{p} w_{i} \cdot\left(x_{z_{0}}-x_{z}\right)+\tilde{G}\left(x_{z}\right),
$$

but

$$
\begin{aligned}
\bigwedge_{i=1}^{p} w_{i} \cdot(x & \left.-x_{z_{0}}\right)+\bigwedge_{i=1}^{p} w_{i} \cdot\left(x_{z_{0}}-x_{z}\right) \\
& =\lambda \bigwedge_{i=1}^{p} w_{i} \cdot\left(x-x_{z}\right)+(1-\lambda) \bigwedge_{i=1}^{p} w_{i} \cdot\left(x-x_{z}\right)
\end{aligned}
$$

so that

$$
\bigwedge_{i=1}^{p} w_{i} \cdot\left(x-x_{z_{0}}\right)+\bigwedge_{i=1}^{p} w_{i} \cdot\left(x_{z_{0}}-x_{z}\right)=\bigwedge_{i=1}^{p} w_{i} \cdot\left(x-x_{z}\right)
$$

Hence

$$
\bigwedge_{i=1}^{p} w_{i} \cdot\left(x-x_{z_{0}}\right)+\tilde{G}\left(x_{z_{0}}\right) \geq \bigwedge_{i=1}^{p} w_{i} \cdot\left(x-x_{z}\right)+\tilde{G}\left(x_{z}\right)
$$

Replacing the above infima by suprema and using the second inequality in Lemma 2.1, we obtain the result for the function $V_{h}$.

We will also need the following lemma.

LEMMA 2.3. If G satisfies (1.7),

$$
G(x)-C^{*} h^{1 / 2} \leq \Lambda_{h}(x) \leq G(x) \leq V_{h}(x) \leq G(x)+C^{*} h^{1 / 2} \quad \forall x \in \Omega,
$$

where $C^{*}=2 q \max _{i}\left\|w_{i}\right\| \max _{i}\left\|v_{i}\right\|(\|\cdot\|$ denotes the Euclidian norm).

Proof. Let $z \in \mathbb{Z}^{q}$ and $x \in \Omega$. Using Lemma 2.1, one has

$$
\bigwedge_{i=1}^{p} w_{i} \cdot\left(x-x_{z}\right)+\tilde{G}\left(x_{z}\right) \leq G(x) \quad \forall x_{z}
$$

so that

$$
\Lambda_{h}(x) \leq G(x)
$$


542 On some nonconvex obstacle problems

Now we denote by $x^{\prime}$ the component of $x$ on $P_{W}(\Omega)$, the orthogonal projection of $\Omega$ onto $W$. There exists $z_{0}$ such that $x^{\prime} \in C_{z_{0}}$, then $x^{\prime}$ can be written as follows:

$$
x^{\prime}=x_{z_{0}}+\sum_{i=1}^{q} \beta_{i} h^{1 / 2} v_{i}, \quad \beta_{i} \in[0,1]
$$

Hence

$$
\left\|x^{\prime}-x_{z_{0}}\right\| \leq q \max _{i}\left\|v_{i}\right\| h^{1 / 2}
$$

so that

$$
\begin{aligned}
\left|\bigwedge_{i=1}^{p} w_{i} \cdot\left(x-x_{z_{0}}\right)\right| & =\left|\bigwedge_{i=1}^{p} w_{i} \cdot\left(x^{\prime}-x_{z_{0}}\right)\right| \\
& \leq q \max _{i}\left\|w_{i}\right\| \max _{i}\left\|v_{i}\right\| h^{1 / 2} .
\end{aligned}
$$

Since

$$
\Lambda_{h}(x) \geq \bigwedge_{i=1}^{p} w_{i} \cdot\left(x-x_{z_{0}}\right)+\tilde{G}\left(x_{z_{0}}\right)
$$

one gets

$$
\Lambda_{h}(x) \geq-q \max _{i}\left\|w_{i}\right\| \max _{i}\left\|v_{i}\right\| h^{1 / 2}+\tilde{G}\left(x_{z_{0}}\right) .
$$

Since

$$
\tilde{G}\left(x_{z_{0}}\right)=\tilde{G}\left(x_{z_{0}}\right)-G(x)+G(x) \geq \bigwedge_{i=1}^{p} w_{i} \cdot\left(x_{z_{0}}-x\right)+G(x),
$$

one obtains by (2.30)

$$
\Lambda_{h}(x) \geq-2 q \max _{i}\left\|w_{i}\right\| \max _{i}\left\|v_{i}\right\| h^{1 / 2}+G(x) .
$$

Replacing again the above infima by suprema, one easily obtains the remaining inequalities. This completes the proof of the lemma.

Remark 2.4. We have seen that condition (1.7) implies (2.3). The two conditions are actually equivalent. Indeed, if (2.3) is verified, then, due to (2.25), the sequence $\left(\Lambda_{h}\right)$ converges uniformly to $G$. Since $\nabla \Lambda_{h}(x)=w_{i}$ 
almost everywhere in $\Omega$, one has at least for a subsequence that $\nabla \Lambda_{h} \rightarrow$ $\nabla G$ in $L^{\infty}(\Omega)$ weak *. Let $B$ be any ball included in $\Omega$. Since

$$
\frac{1}{|B|} \int_{B} \nabla \Lambda_{h}(x) d x \in \operatorname{Co}\left(w_{i}\right)
$$

and $\operatorname{Co}\left(w_{i}\right)$ is a closed set, one has

$$
\frac{1}{|B|} \int_{B} \nabla G(x) d x \in \operatorname{Co}\left(w_{i}\right)
$$

Using the Lebesgue differentiation theorem, one obtains (1.7).

\section{Statement and proof of the main results}

First we assume that the obstacles do not touch each other, that is,

$$
\alpha<\beta \quad \text { in } \bar{\Omega} \text {. }
$$

Thus the following constants are positive:

$$
M_{1}:=\inf _{x \in \Omega_{1}}(G(x)-\alpha(x)), \quad M_{2}:=\inf _{x \in \Omega_{2}}(\beta(x)-G(x)),
$$

where $\Omega_{1}$ and $\Omega_{2}$ denote the following sets:

$$
\Omega_{1}:=\left\{x \in \Omega: G(x)>\frac{\alpha(x)+\beta(x)}{2}\right\}, \quad \Omega_{2}:=\Omega \backslash \Omega_{1} .
$$

Then one has the following theorem.

THEOREM 3.1. Assume that $\varphi$ is a continuous function satisfying (1.1) and (1.2). If (1.7) holds, then

$$
I:=\inf _{u \in \mathcal{K}} \int_{\Omega} \varphi(\nabla u(x)) d x=0
$$

Moreover, if $\Omega$ is polyhedral, then, under the assumptions of Lemma 2.2, there exists a constant $C$ independent of $h, 0<h<\inf \left(\left(M_{1} / C^{*}\right)^{2},\left(M_{2} / C^{*}\right)^{2}, 1\right)$ such that

$$
I:=\inf _{u \in \mathcal{K}_{h}} \int_{\Omega} \varphi(\nabla u(x)) d x \leq C h^{1 / 2},
$$

where $C^{*}=2 q \max _{i}\left\|w_{i}\right\| \max _{i}\left\|v_{i}\right\|$. 
544 On some nonconvex obstacle problems

Proof. We consider the following Lipschitz functions:

$$
\begin{aligned}
& u_{1}^{h}(x)=\Lambda_{h} \vee\left(G(x)-\operatorname{dist}\left(x, \partial \Omega_{1}\right)\right) \quad \text { if } x \in \Omega_{1}, \\
& u_{2}^{h}(x)=V_{h}(x) \wedge\left(G(x)+\operatorname{dist}\left(x, \partial \Omega_{2}\right)\right) \quad \text { if } x \in \Omega_{2},
\end{aligned}
$$

where $\operatorname{dist}\left(x, \partial \Omega_{i}\right)$ denotes the distance from $x$ to the boundary $\partial \Omega_{i}$ of $\Omega_{i}, i=1,2$. The functions $\Lambda_{h}$ and $V_{h}$ are defined by (2.14). Then one has

$$
\begin{array}{ll}
\alpha(x) \leq u_{1}^{h}(x) \leq G(x) & \text { in } \Omega_{1}, \\
G(x) \leq u_{2}^{h}(x) \leq \beta(x) & \text { in } \Omega_{2} .
\end{array}
$$

We prove (3.7); (3.8) can be proved the same way. According to Lemma 2.3, one has

$$
u_{1}^{h}(x) \leq G(x) \quad \forall x \in \Omega_{1} .
$$

On the other hand, one has

$$
\begin{aligned}
u_{1}^{h}(x) & \geq\left(G(x)-C^{*} h^{1 / 2}\right) \vee\left(G(x)-\operatorname{dist}\left(x, \partial \Omega_{1}\right)\right) \\
& \geq G(x)-C^{*} h^{1 / 2}
\end{aligned}
$$

Thus

$$
u_{1}^{h}(x) \geq G(x)-\alpha(x)-C^{*} h^{1 / 2}+\alpha(x)
$$

so that

$$
u_{1}^{h}(x) \geq M_{1}-C^{*} h^{1 / 2}+\alpha(x),
$$

where $M_{1}$ is defined by (3.2). Since $0<h<\left(M_{1} / C^{*}\right)^{2}$, one gets

$$
u_{1}^{h}(x) \geq \alpha(x) .
$$

It is clear by (2.25) that the functions $u_{i}^{h \prime}$ s coincide with $G$ at the boundaries of $\Omega_{i}$ 's, respectively. Since $\partial \Omega \subset \partial \Omega_{1} \cup \partial \Omega_{2}$, the function

$$
u_{h}(x)= \begin{cases}u_{1}^{h}(x), & \text { if } x \in \Omega_{1}, \\ u_{2}^{h}(x), & \text { if } x \in \Omega_{2},\end{cases}
$$

coincides with $G$ at the boundary of $\Omega$. Moreover, $u_{h}$ is a Lipschitz function. Indeed, let $x \in \Omega_{1}$ and $y \in \Omega_{2}$. There exists $z \in \partial \Omega_{1} \cap \partial \Omega_{2}$ such that

$$
z \in[x, y]
$$


Thus

$$
\begin{aligned}
\left|u_{h}(x)-u_{h}(y)\right| & =\left|u_{1}^{h}(x)-u_{2}^{h}(y)\right| \\
& \leq\left|u_{1}^{h}(x)-u_{1}^{h}(z)\right|+\left|u_{2}^{h}(z)-u_{2}^{h}(y)\right|
\end{aligned}
$$

since $u_{1}^{h}(z)=u_{2}^{h}(z)=G(z)$. Hence

$$
\left|u_{h}(x)-u_{h}(y)\right| \leq C\{|x-z|+|z-y|\}=C|x-y|,
$$

where $C$ is a constant independent of $x, y$, and $h$ (in the sequel, we will denote by $C$ every constant which does not depend on $h$ ). Then the function $u_{h}$ belongs to $\mathcal{K}$. Now we can prove (3.4). Due to (2.25), one has

$$
\begin{aligned}
& u_{h}=\Lambda_{h} \quad \text { on }\left\{x \in \Omega_{1}: \operatorname{dist}\left(x, \partial \Omega_{1}\right) \geq C^{*} h^{1 / 2}\right\}, \\
& u_{h}=V_{h} \text { on }\left\{x \in \Omega_{2}: \operatorname{dist}\left(x, \partial \Omega_{2}\right) \geq C^{*} h^{1 / 2}\right\} .
\end{aligned}
$$

Therefore, $\nabla u_{h}=w_{i}$ except in a neighborhood $N_{h}$ of the boundaries $\partial \Omega_{i}$ of measure less than $C h^{1 / 2}$. Using (1.1) and the fact that $\nabla u_{h}$ is uniformly bounded, one obtains

$$
0 \leq I=\int_{N_{h}} \varphi\left(\nabla u_{h}(x)\right) d x \leq C h^{1 / 2}
$$

for every $0<h<\inf \left(\left(M_{1} / C^{*}\right)^{2},\left(M_{2} / C^{*}\right)^{2}\right)$, which obviously implies (3.4). Due to (2.25) again, one has

$$
\left|G(x)-\Lambda_{h}(x)\right| \leq C^{*} h^{1 / 2}, \quad\left|G(x)-V_{h}(x)\right| \leq C^{*} h^{1 / 2}, \quad \forall x \in \bar{\Omega},
$$

so that

$$
\left|G(x)-u_{h}(x)\right| \leq C h^{1 / 2} \quad \forall x \in \bar{\Omega} .
$$

Let $\hat{u}_{h}$ denote the interpolate of $u_{h}$. Clearly, $\hat{u}_{h} \in \mathcal{K}_{h}$ and one has

$$
\left|u_{h}(x)-\hat{u}_{h}(x)\right| \leq C h .
$$

We prove (3.22). Let $x \in \Omega$. There exists an $N$-simplex $K \in \tau_{h}$ such that $x \in K$. Let $y$ be any vertex of the $N$-simplex $K$. One has

$$
u_{h}(y)=\hat{u}_{h}(y)
$$

Therefore, one has

$$
\left|u_{h}(x)-\hat{u}_{h}(x)\right| \leq\left|u_{h}(x)-u_{h}(y)\right|+\left|\hat{u}_{h}(x)-\hat{u}_{h}(y)\right|
$$


546 On some nonconvex obstacle problems

Using the mean value theorem, one obtains

$$
\left|u_{h}(x)-\hat{u}_{h}(x)\right| \leq\left\{\sup _{z \in K}\left\|\nabla u_{h}(z)\right\|+\sup _{z \in K}\left\|\nabla \hat{u}_{h}(z)\right\|\right\}\|x-y\| .
$$

Since $\nabla u_{h}$ is uniformly bounded, $\nabla \hat{u}_{h}$ is also uniformly bounded (see [4]). Moreover, $\|x-y\| \leq h_{K} \leq h$. Thus there exists a constant $C$ so that (3.22) holds. Therefore, using the triangle inequality (recall that $h<1$ ), one gets

$$
\left|\hat{u}_{h}(x)-G(x)\right| \leq C h^{1 / 2} .
$$

Notice that

$$
\nabla \hat{u}_{h}=w_{i}
$$

except maybe on the set $S$ composed of simplices where interpolation occurred. Since on this set $\nabla \hat{u}_{h}$ remains bounded, one has

$$
\int_{\Omega} \varphi\left(\nabla \hat{u}_{h}(x)\right) d x=\int_{S} \varphi\left(\nabla \hat{u}_{h}(x)\right) d x \leq C|S|,
$$

where $|S|$ is the Lebesgue measure of $S$. When

$$
\operatorname{dist}\left(x, \partial \Omega_{1} \cup \partial \Omega_{2}\right) \geq C^{*} h^{1 / 2},
$$

one has

$$
u_{h}(x)=\Lambda_{h}(x) \text { or } V_{h} .
$$

So, in the set where $\operatorname{dist}\left(x, \partial \Omega_{1} \cup \partial \Omega_{2}\right) \geq C^{*} h^{1 / 2}+h$, one has that $\hat{u}_{h}$ equals the interpolate of $\Lambda_{h}$ or $V_{h}$. We denote by $S_{1}$ the set

$$
S_{1}=\left\{x \in \Omega: \operatorname{dist}\left(x, \partial \Omega_{1} \cup \partial \Omega_{2}\right) \geq C^{*} h^{1 / 2}+h\right\} .
$$

Hence

$$
|S| \leq\left|S \cap S_{1}\right|+\left|\Omega \backslash S_{1}\right| .
$$

First we have

$$
\left|\Omega \backslash S_{1}\right| \leq C h^{1 / 2} .
$$

To estimate $\left|S \cap S_{1}\right|$, one sees that interpolation occurs on an $h$-neighborhood of the set where $\Lambda_{h}$ and $V_{h}$ have discontinuity in their gradients. 
Clearly, $\Lambda_{h}$ and $V_{h}$ have a jump in their gradients on a unit cell of the lattice spanned by $h^{1 / 2} v_{i}$ 's when one of the functions

$$
w_{i} \cdot\left(x-x_{z}\right)+\tilde{G}\left(x_{z}\right)
$$

is equal to another. These two functions are equal on a set of $(q-1)$ dimensional measure bounded by $C h^{(q-1) / 2}$. Since in Lemma 2.2 the supremum and infimum are taken on a finite number of functions, it is clear that

$$
\left|S \cap S_{1}\right| \leq C h^{(q-1) / 2} \cdot h \cdot N(h)
$$

where $N(h)$ is the number of cells of size $h^{1 / 2}$ included in $P_{W}(\Omega)$. Clearly, $N(h) h^{q / 2}$ is less than or equal to the $q$-dimensional measure of $P_{W}(\Omega)$. Therefore,

$$
\left|S \cap S_{1}\right| \leq C h^{1 / 2}
$$

Combining (3.28), (3.33), and (3.36), one obtains

$$
\int_{\Omega} \varphi\left(\nabla \hat{u}_{h}\right) d x \leq C h^{1 / 2} .
$$

This completes the proof of the theorem.

Remark 3.2. We chose the cells $C_{z}$ 's of size $h^{1 / 2}$ among those of size $h^{\alpha}$, $\alpha \in(0,1)$, since they provide the best estimate (see [6] for details).

Remark 3.3. Notice that (3.4) is still valid if $\Omega$ is the union of a finite number of convex domains. Moreover, a polyhedral domain can be divided into a finite number of disjoint convex polyhedral domains. Using the same construction as above in every such subdomain, one can see that the estimate obtained in Theorem 3.1 is obviously still valid.

Remark 3.4. The continuous problem (1.6) does not admit in general a minimizer. Indeed, we assume that $q<n$ and

$$
\nabla G \neq w_{i} \quad \text { in a set of positive measure. }
$$

There exists $v \in \mathbb{R}^{n}$ such that

$$
w_{i} \cdot v=0 \quad \forall i=1,2, \ldots, p
$$

If problem (1.6) admits a minimizer $u$, by (3.4), (1.1), and (1.2), one has

$$
\nabla u=w_{i} \quad \text { a.e. in } \Omega \text {. }
$$


548 On some nonconvex obstacle problems

Using the variant of Poincaré's inequality

$$
\int_{\Omega}|u(x)-G(x)| d x \leq C \int_{\Omega}|(\nabla u(x)-\nabla G(x)) \cdot v| d x
$$

(1.7), (3.39), and (3.40), one deduces that

$$
u=G \text {. }
$$

But the assertions (3.38) and (3.40) are incompatible. Therefore, problem (1.6) cannot admit a minimizer. On the other hand and by a compactness argument, the discrete problem $I_{h}$ admits minimizers.

We end this paper by considering the case where the two obstacles touch each other. We denote by $\Omega^{\prime}$ the following open set:

$$
\Omega^{\prime}:=\{x \in \Omega: \alpha(x)<\beta(x)\} .
$$

One has, for every $u \in \mathcal{K}$,

$$
\begin{gathered}
u(x)=G(x)=\alpha(x)=\beta(x) \quad \text { in } \Omega \backslash \Omega^{\prime} \\
\int_{\Omega} \varphi(\nabla u(x)) d x=\int_{\Omega^{\prime}} \varphi(\nabla u(x)) d x+\int_{\Omega \backslash \Omega^{\prime}} \varphi(\nabla G(x)) d x
\end{gathered}
$$

Since

$$
u=G \quad \text { on } \partial \Omega^{\prime},
$$

one has

$$
\int_{\Omega} \varphi(\nabla u(x)) d x \geq \inf _{v \in \mathcal{K}\left(\Omega^{\prime}\right)} \int_{\Omega^{\prime}} \varphi(\nabla v(x)) d x+\int_{\Omega \backslash \Omega^{\prime}} \varphi(\nabla G(x)) d x .
$$

Therefore,

$$
\inf _{v \in \mathcal{K}(\Omega)} \int_{\Omega} \varphi(\nabla v(x)) d x \geq \inf _{v \in \mathcal{K}\left(\Omega^{\prime}\right)} \int_{\Omega^{\prime}} \varphi(\nabla v(x)) d x+\int_{\Omega \backslash \Omega^{\prime}} \varphi(\nabla G(x)) d x
$$

Now let $u \in \mathcal{K}\left(\Omega^{\prime}\right)$. We define $\tilde{u} \in \mathcal{K}(\Omega)$ as follows:

$$
\tilde{u}=u \quad \text { in } \Omega^{\prime}, \quad \tilde{u}=G \quad \text { in } \Omega \backslash \Omega^{\prime} .
$$


Then

$$
\begin{aligned}
\inf _{v \in \mathcal{K}(\Omega)} \int_{\Omega} \varphi(\nabla v(x)) d x & \leq \int_{\Omega} \varphi(\nabla \tilde{u}(x)) d x \\
& =\int_{\Omega^{\prime}} \varphi(\nabla u(x)) d x+\int_{\Omega \backslash \Omega^{\prime}} \varphi(\nabla G(x)) d x .
\end{aligned}
$$

Therefore,

$$
\inf _{v \in \mathcal{K}(\Omega)} \int_{\Omega} \varphi(\nabla v(x)) d x=\inf _{v \in \mathcal{K}\left(\Omega^{\prime}\right)} \int_{\Omega^{\prime}} \varphi(\nabla v(x)) d x+\int_{\Omega \backslash \Omega^{\prime}} \varphi(\nabla G(x)) d x .
$$

Then one has the following theorem.

THEOREM 3.5. Assume that $\varphi$ is a continuous function satisfying (1.1) and (1.2). If (1.7) holds,

$$
\inf _{u \in \mathcal{K}(\Omega)} \int_{\Omega} \varphi(\nabla u(x)) d x=\int_{\Omega \backslash \Omega^{\prime}} \varphi(\nabla G(x)) d x .
$$

Proof. Due to (3.51), it suffices to prove that

$$
\inf _{u \in \mathcal{K}\left(\Omega^{\prime}\right)} \int_{\Omega^{\prime}} \varphi(\nabla u(x)) d x=0 .
$$

We denote by $\Omega_{\varepsilon}^{\prime}$ the following set:

$$
\Omega_{\varepsilon}^{\prime}=\left\{x \in \Omega^{\prime}: \operatorname{dist}\left(x, \partial \Omega^{\prime}\right)>\varepsilon\right\} .
$$

Let $u \in \mathcal{K}\left(\Omega_{\varepsilon}^{\prime}\right)$. We extend $u$ to $\Omega^{\prime}$ by setting

$$
u=G \quad \text { in } \Omega^{\prime} \backslash \Omega_{\varepsilon}^{\prime} .
$$

The extension of $u$ to $\Omega^{\prime}$ belongs to $\mathcal{K}\left(\Omega^{\prime}\right)$ and

$$
\int_{\Omega^{\prime}} \varphi(\nabla u(x)) d x=\int_{\Omega_{\varepsilon}^{\prime}} \varphi(\nabla u(x)) d x+\int_{\Omega^{\prime} \backslash \Omega_{\varepsilon}^{\prime}} \varphi(\nabla G(x)) d x .
$$

Thus

$$
\int_{\Omega_{\varepsilon}^{\prime}} \varphi(\nabla u(x)) d x+\int_{\Omega^{\prime} \backslash \Omega_{\varepsilon}^{\prime}} \varphi(\nabla G(x)) d x \geq \inf _{v \in \mathcal{K}\left(\Omega^{\prime}\right)} \int_{\Omega^{\prime}} \varphi(\nabla v(x)) d x,
$$


which implies that

$$
\inf _{v \in \mathcal{K}\left(\Omega^{\prime}\right)} \int_{\Omega^{\prime}} \varphi(\nabla v(x)) d x \leq \inf _{v \in \mathcal{K}\left(\Omega_{\varepsilon}^{\prime}\right)} \int_{\Omega_{\varepsilon}^{\prime}} \varphi(\nabla v(x)) d x+\int_{\Omega^{\prime} \backslash \Omega_{\varepsilon}^{\prime}} \varphi(\nabla G(x)) d x .
$$

But we know that

$$
\inf _{v \in \mathcal{K}\left(\Omega_{\varepsilon}^{\prime}\right)} \int_{\Omega_{\varepsilon}^{\prime}} \varphi(\nabla v(x)) d x=0
$$

since in the set $\Omega_{\varepsilon}$ the obstacles do not touch each other. Thus

$$
0 \leq \inf _{v \in \mathcal{K}\left(\Omega^{\prime}\right)} \int_{\Omega^{\prime}} \varphi(\nabla v(x)) d x \leq C^{\prime}\left|\Omega^{\prime} \backslash \Omega_{\varepsilon}^{\prime}\right| \leq C^{\prime \prime} \varepsilon,
$$

where $C^{\prime}$ and $C^{\prime \prime}$ are constants independent of $\varepsilon$. Hence,

$$
\inf _{v \in \mathcal{K}\left(\Omega^{\prime}\right)} \int_{\Omega^{\prime}} \varphi(\nabla v(x)) d x=0,
$$

and this completes the proof of the theorem.

Remark 3.6. Using (3.45), one should notice that if $u$ is a minimizer of

$$
\inf _{v \in \mathcal{K}(\Omega)} \int_{\Omega} \varphi(\nabla v(x)) d x
$$

then the restriction of $u$ to $\Omega^{\prime}$ is also a minimizer of

$$
\inf _{v \in \mathcal{K}\left(\Omega^{\prime}\right)} \int_{\Omega^{\prime}} \varphi(\nabla v(x)) d x
$$

Therefore, the continuous problem (1.6) cannot admit a minimizer in general. Moreover, if $\nabla G(x) \neq w_{i}$ in a subset of $\Omega \backslash \Omega^{\prime}$ of positive measure, one has

$$
I=\inf _{v \in \mathcal{K}(\Omega)} \int_{\Omega} \varphi(\nabla v(x)) d x=\int_{\Omega \backslash \Omega^{\prime}} \varphi(\nabla G(x)) d x>0 .
$$

\section{Acknowledgments}

I would like to thank B. Brighi and M. Fuchs for helpful discussions regarding this paper. I would also like to thank the referee for useful comments which helped to improve the readability of this work. 


\section{References}

[1] R. A. Adams, Sobolev Spaces, Academic Press, New York, 1975.

[2] J. M. Ball and R. D. James, Fine phase mixtures as minimizers of energy, Arch. Rational Mech. Anal. 100 (1987), no. 1, 13-52.

[3] , Proposed experimental tests of a theory of fine microstructure and the twowell problem, Philos. Trans. Roy. Soc. London Ser. A 338 (1992), 389-450.

[4] B. Brighi and M. Chipot, Approximated convex envelope of a function, SIAM J. Numer. Anal. 31 (1994), no. 1, 128-148.

[5] M. Chipot, Energy estimates for variational problems with nonhomogeneous boundary conditions, Nonlinear Mathematical Problems in Industry, II (Iwaki, 1992), GAKUTO Internat. Ser. Math. Sci. Appl., vol. 2, Gakkōtosho, Tokyo, 1993, pp. 473-487.

[6] M. Chipot and A. Elfanni, On the numerical analysis of some variational problems with nonhomogeneous boundary conditions, Japan J. Indust. Appl. Math. 15 (1998), no. 3, 345-361.

[7] M. Chipot and W. Li, Variational problems with potential wells and nonhomogeneous boundary conditions, Calculus of Variations, Homogenization and Continuum Mechanics (Marseille, 1993) (G. Bouchitté, G. Buttazzo, and P. Suquet, eds.), Ser. Adv. Math. Appl. Sci., vol. 18, World Scientific Publishing, New Jersey, 1994, pp. 149-168.

[8] A. Elfanni, Sur quelques questions d'analyse numérique relatives à des problèmes non convexes, Ph.D. thesis, University of Metz, France, 1996.

[9] _ On some variational problems with an infinite number of wells, Elliptic and Parabolic Problems (Rolduc/Gaeta, 2001), World Scientific Publishing, New Jersey, 2002, pp. 66-74.

[10] G. Friesecke, A necessary and sufficient condition for nonattainment and formation of microstructure almost everywhere in scalar variational problems, Proc. Roy. Soc. Edinburgh Sect. A 124 (1994), no. 3, 437-471.

[11] P.-A. Raviart and J.-M. Thomas, Introduction à l'analyse numérique des équations aux dérivées partielles, Collection Mathématiques Appliquées pour la Maîtrise, Masson, Paris, 1983.

A. Elfanni: Fachbereich 6.1-Mathematik, Universität des Saarlandes, Postfach

1511 50, 66041 Saarbrücken, Germany

E-mail address: elfanni@math.uni-sb.de 


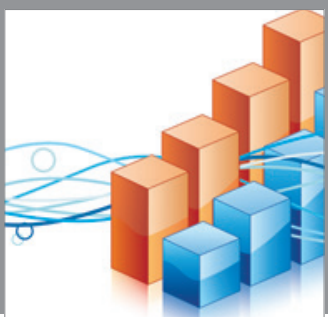

Advances in

Operations Research

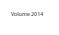

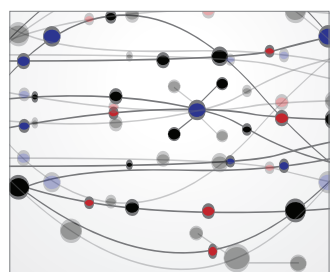

\section{The Scientific} World Journal
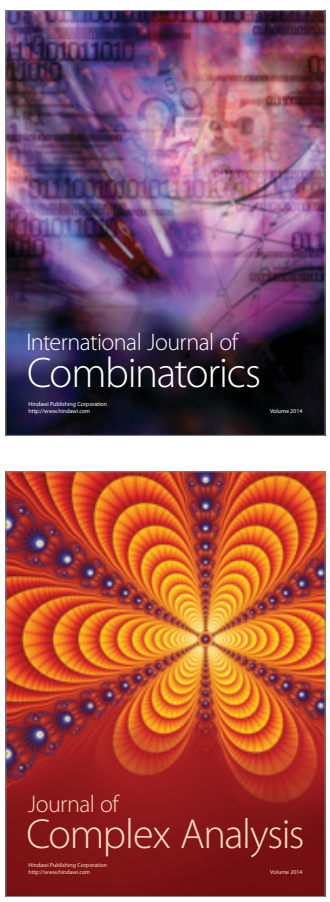

International Journal of

Mathematics and

Mathematical

Sciences
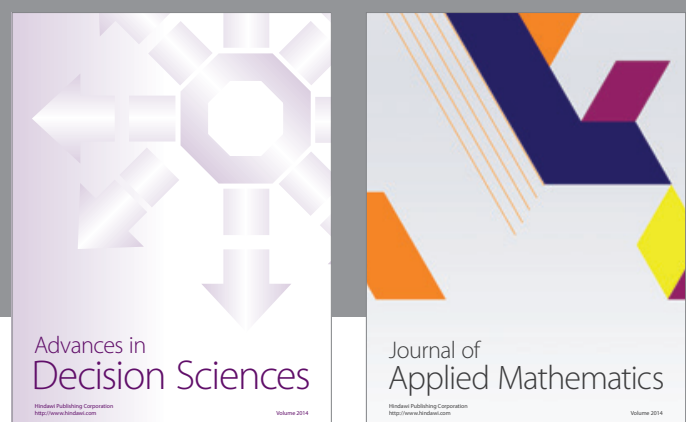

Journal of

Applied Mathematics
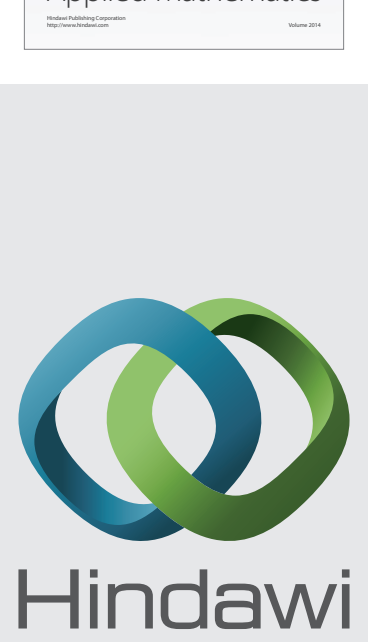

Submit your manuscripts at http://www.hindawi.com
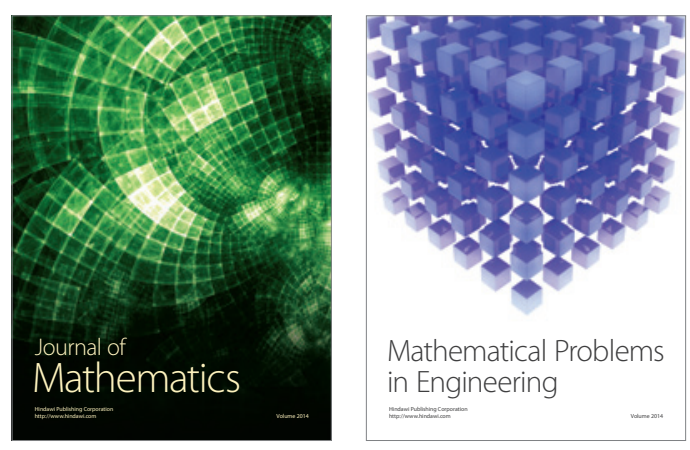

Mathematical Problems in Engineering
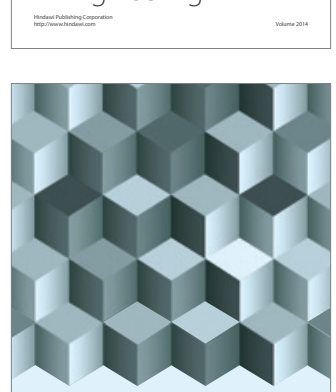

Journal of

Function Spaces
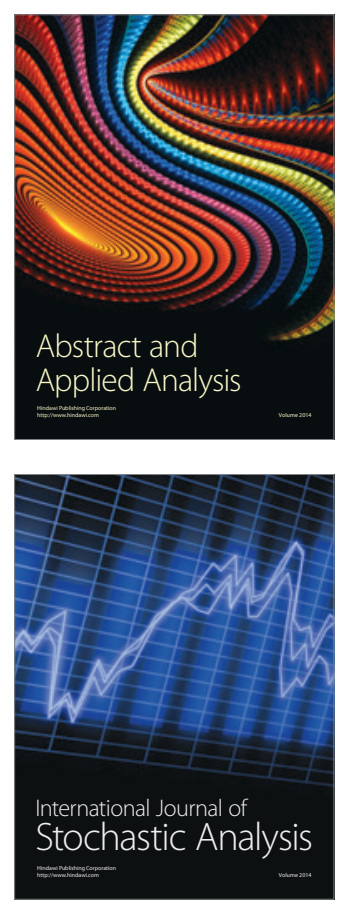

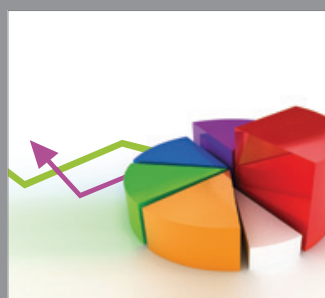

ournal of

Probability and Statistics

Promensencen
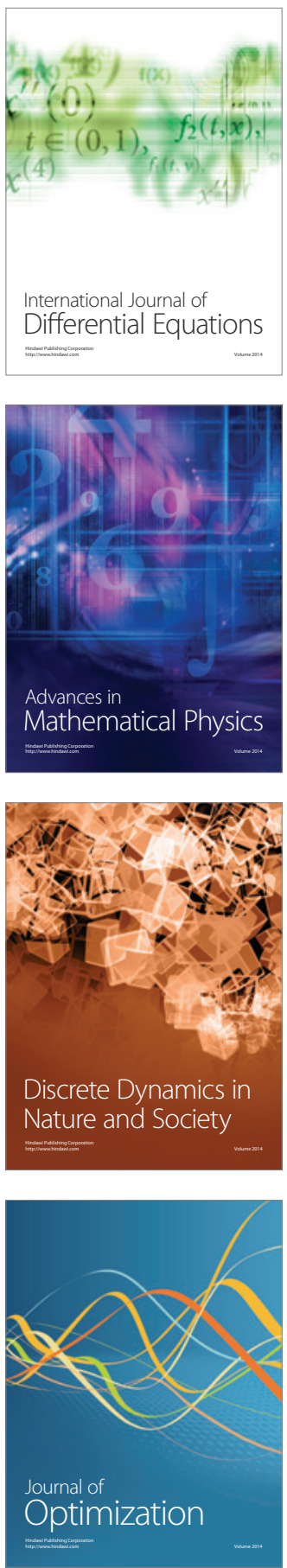